\title{
Effect of Host-Related Factors on the Intensity of Liver Fibrosis in Patients With Chronic Hepatitis C Virus Infection
}

\author{
Luciano Bello Costa, Maria Lucia Gomes Ferraz, \\ Renata M. Perez, Adalgisa S. Ferreira, \\ Carla Adriana L. Matos, Valéria P. Lanzoni and \\ Antônio Eduardo Silva
}

\author{
Department of Gastroenterology, Medical \\ School of Medicine of São Paulo, UNIFESP, \\ São Paulo, SP, Brazil
}

\begin{abstract}
There is increasing interest in the identification of factors associated with liver disease progression in patients infected with hepatitis $\mathrm{C}$ virus $(\mathrm{HCV})$. We assessed host-related factors associated with a histologically advanced stage of this disease and determined the rate of liver fibrosis progression in $\mathrm{HCV}$-infected patients. We included patients submitted to liver biopsy, who were anti-HCV and HCV RNA positive, who showed a parenteral risk factor (blood transfusion or intravenous drug use), and who gave information about alcohol consumption.Patients were divided into two groups for analysis: group 1 - grades 0 to 2; group 2 - grades 3 to 4 . The groups were compared in terms of sex, age at the time of infection, estimated duration of infection and alcoholism. The rate of fibrosis progression (index of fibrosis) was determined based on the relationship between disease stage and duration of infection (years). Logistic regression analysis revealed that age at the time of infection $(\mathrm{P}<0.01 ; 95 \% \mathrm{CI} 1.06-1.22)$ and the duration of infection $(\mathbf{P}<0.01 ; 95 \%$ CI 1.06-1.32) were independently associated with a more advanced stage of hepatitis $\mathrm{C}$. The median index of fibrosis was 0.14 for the group as a whole. A significant difference in the index of fibrosis was observed between patients aged $<40$ years at infection $($ median $=0.11)$ and patients aged $\geq 40$ years $($ median $=0.47$ ). The main factors associated with a more rapid fibrosis progression were age at the time of infection and the estimated duration of infection. Patients who acquired $\mathrm{HCV}$ after 40 years of age showed a higher rate of fibrosis progression.
\end{abstract}

Key Words: Hepatitis C virus, liver fibrosis, patients.

Hepatitis $\mathrm{C}$ virus (HCV) infection affects people worldwide and about 170 million individuals are estimated to be chronically infected with HCV. Several aspects of the natural history of this disease are still not completely understood.

After acute infection, viremia persists in $85 \%$ of the patients [1] and most of them develop chronic hepatitis. Different studies have shown variable rates of progression to cirrhosis in patients with chronic hepatitis $\mathrm{C}$ according to the group analyzed. Only $2 \%$ of women

Received on 03 March 2002; revised 14 September 2002. Address for correspondence: Dr. Maria Lucia Gomes Ferraz Rua Machado Bittencourt 413, apto 81, Zip code: 04044-001, São Paulo - SP, Brazil.

The Brazilian Journal of Infectious Diseases 2002;6(5):219-224 (C) 2002 by The Brazilian Journal of Infectious Diseases and Contexto Publishing. All rights reserved. $1413-8670$ contaminated through the use of immunoglobulin evolved to cirrhosis over a follow-up period of 17 years [2], while cirrhosis was observed in $51 \%$ of patients contaminated through blood transfusion [3].

During cirrhosis, patients may develop complications secondary to portal hypertension and/or hepatic insufficiency; they may also have a higher risk for hepatocellular carcinoma ( 2 to $4 \%$ per year) $[4,5]$. These complications are intimately related to the degree of fibrosis and destructuring of the lobular architecture and, therefore, staging represents the main prognostic marker in patients with chronic hepatitis $C$.

Knowledge about the factors that influence the natural history of hepatitis $\mathrm{C}$ is important for the clinical treatment of patients and for the implementation of preventive and therapeutic measures.

Our objective was to assess the effect of hostrelated factors on the histological manifestations of 
chronic hepatitis $\mathrm{C}$ and to determine the rate of liver fibrosis progression in patients chronically infected with HCV.

\section{Material and Methods}

Data were obtained from the medical records of patients seen at the Hepatitis Outpatient Clinic of São Paulo Hospital, Escola Paulista de Medicina, from 1994 to 2000.

$\mathrm{HCV}$-infected patients who met the following criteria were included in the study: patients who tested positive for HCV RNA, who showed a parenteral risk factor (blood transfusion and/or intravenous drug use), who reported information about alcohol consumption, and for whom a liver biopsy adequate for analysis was available.

Only patients who could provide information about the date of blood transfusion and/or intravenous drug use were included. The duration of infection was estimated based on the date of the first blood transfusion or the first year of intravenous drug use, with either occurrence being considered the probable date of infection.

Alcohol consumption was determined as grams of ethanol per day, and only patients for whom sufficient data for the calculation of daily ethanol consumption were available were included.

A liver biopsy was considered to be adequate for analysis when it provided a representative fragment for histological evaluation. Staging was based on the grade of fibrosis and destructuring of the lobular architecture, and stages were classified according to the semiquantitative criteria proposed by Ludwig and Desmet [6, 7] for the classification of chronic hepatitis: 0 - absence of fibrosis, 1 - portal fibrosis, 2 - portalportal septa with preserved lobular architecture, 3 portal-portal and portal-central septa, with the presence of nodules, but preserved areas of lobular architecture, and 4 - cirrhosis.

Patients co-infected with hepatitis B virus and/or HIV, patients with chronic renal failure, immunosuppressed patients, and patients with a history of previous treatment were excluded from the study.
For analysis of factors associated with a more advanced stage of the disease, patients were divided into two groups: group 1 consisted of patients in stage 0,1 or 2 of the disease, and group 2 consisted of patients in stage 3 or 4 . Groups were compared regarding sex, age at the time of infection, estimated duration of infection and alcoholism. Patients were considered to be alcoholics when they reported an ethanol consumption higher than $50 \mathrm{~g}$ per day.

The index of fibrosis was determined by dividing disease stage ( 0 to 4 ) by duration of infection (years) in order to estimate the mean time of progression to cirrhosis among patients chronically infected with $\mathrm{HCV}$.

The chi-square test, Student $t$-test and MannWhitney test were used for statistical analysis. Variables that provided a $\mathrm{P}$ value $<0.20$ upon bivariate analysis were submitted to logistic regression analysis for the identification of variables independently associated with a more advanced stage.

\section{Results}

A total of 153 patients with a history of blood transfusion or intravenous drug use were initially evaluated, and 94 patients who did not meet the criteria described above were excluded. Exclusion criteria were the lack of information about the probable date of infection in 40 patients, insufficient information for the quantification of alcohol consumption in 37 , and an inadequate liver biopsy fragment in 17.

Fifty-nine patients (Table 1), 43 (73\%) men and 16 (27\%) women, were included in the study. The mean age at the time of infection was $29 \pm 14$ years.Fortythree $(73 \%)$ patients had a history of blood transfusion and $16(27 \%)$ had a history of intravenous drug use. The mean duration of infection was $14 \pm 7$ years. Twenty-two (37\%) patients reported an ethanol consumption higher than $50 \mathrm{~g} /$ day and were classified as alcoholics.

The distribution of the patients according to the stage of chronic hepatitis is shown in Figure 1. Thirty-six (61\%) patients were in stages 0 to 2 and were included in group 1; group 2 consisted of 23 (39\%) patients in stage 3 or 4 . 
Table 1. Characteristics of the patients $(n=59)$

\begin{tabular}{lc}
\hline Characteristic & Results \\
\hline Male & $43(73 \%)$ \\
Mean duration of infection (years) & $14 \pm 7$ \\
Mean age at the time of infection (years) & $29 \pm 14$ \\
History of blood transfusion & $43(73 \%)$ \\
History of intravenous drug use & $16(27 \%)$ \\
Alcoholism (ethanol $>$ 50 g/day) & $22(37 \%)$ \\
Grade 3-4 hepatitis C & $23(39 \%)$ \\
\hline
\end{tabular}

Table 2. Comparison between groups

\begin{tabular}{lccr}
\hline & $\begin{array}{c}\text { Group 1 } \\
(\mathbf{n = 3 9 )}\end{array}$ & $\begin{array}{c}\text { Group 2 } \\
(\mathbf{n = 2 3})\end{array}$ & P \\
\hline Male & $75 \%$ & $70 \%$ & 0.65 \\
Mean age at infection (years) & $24 \pm 11$ & $37 \pm 15$ & 0.001 \\
Mean duration of infection (years) & $13 \pm 7$ & $15 \pm 8$ & 0.20 \\
Alcoholism & $39 \%$ & $35 \%$ & 0.75 \\
\hline
\end{tabular}

Table 3. Logistic regression analysis (final model)

\begin{tabular}{lrcc}
\hline & P & OR & $\mathbf{9 5 \% ~ C I ~}$ \\
\hline Age at infection & $<0.001$ & 1.14 & $1.06-1.22$ \\
Duration of infection & 0.003 & 1.18 & $1.06-1.32$ \\
\hline
\end{tabular}

No significant difference in gender $(75 \%$ males in group 1 and $70 \%$ in group $2 ; \mathrm{P}=0.65$ ) or alcoholism (39\% alcoholics in group 1 and $35 \%$ in group 2; $\mathrm{P}=0.75$ ) was observed between groups (Table 2). Mean duration of infection was $13 \pm 7$ years for group 1 and $15 \pm 8$ years for group $2(\mathrm{P}=0.20)$. Mean age at the time of infection was $24 \pm 11$ years for group 1 and $37 \pm 15$ years for group $2(\mathrm{P}=0.001)$.

Logistic regression analysis demonstrated that age at the time of infection ( $\mathrm{P}<0.01,95 \% \mathrm{CI} 1.06-1.22)$ and duration of infection $(\mathrm{P}<0.01,95 \%$ CI 1.06-1.32) were independently associated with a more advanced stage of the disease (Table 3 ).

The median index of fibrosis was 0.14 for the group as a whole.

Since age at the time of infection was identified as a factor independently associated with a more advanced stage, the index of fibrosis was determined according to age at infection $(<40$ and $\geq 40$ years). The median index of fibrosis among patients aged $<40$ years at infection was 0.11 , while this index was 0.47 among patients 
Figure 1. Patient distribution of patients according to disease stage

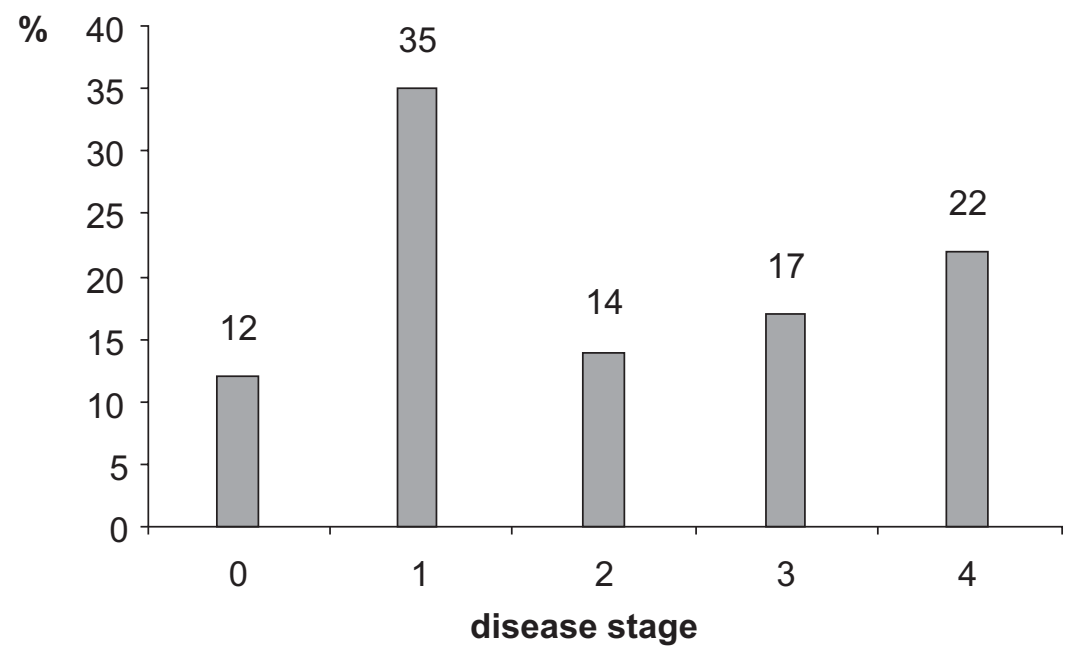

Figure 2. Mean time of progression to cirrhosis according to age at the time of infection

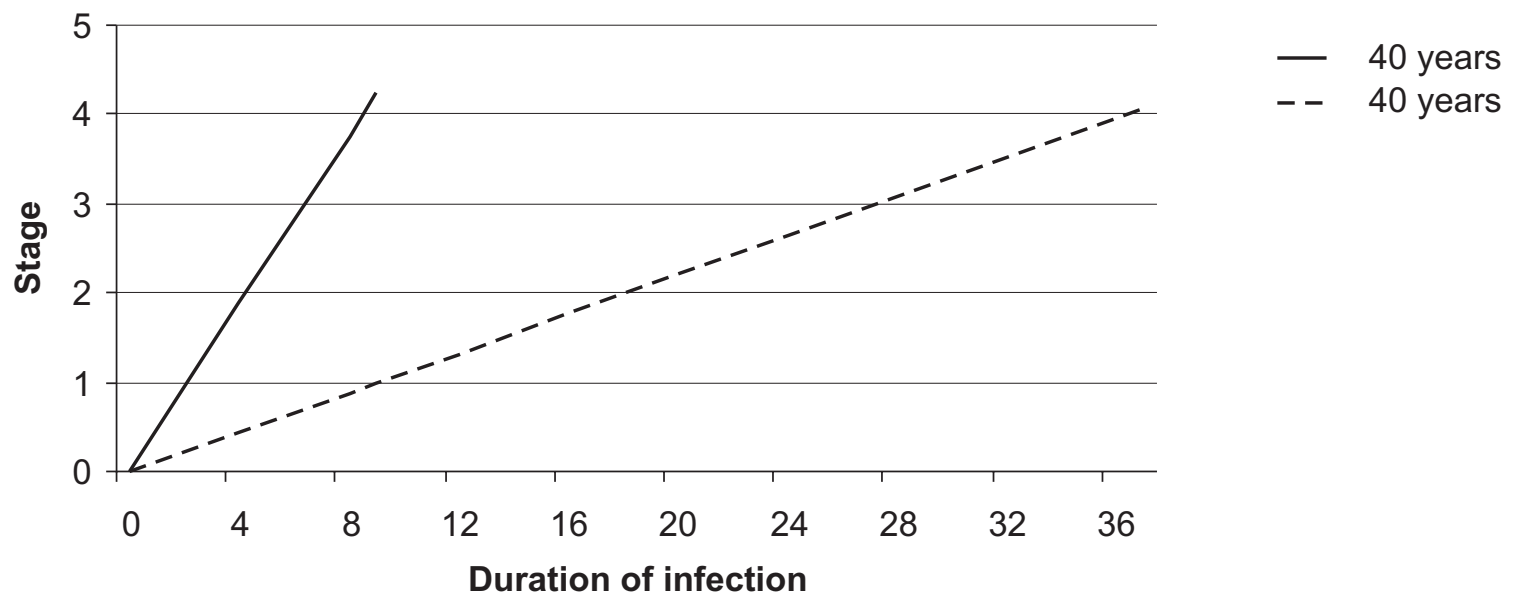


who contracted the infection after 40 years; this difference was found to be significant $(\mathrm{P}<0.001)$.

\section{Discussion}

The only host-related factors showing an independent correlation with the stage of chronic hepatitis were age at the time of infection and duration of infection.

No significant difference in gender or alcoholism was observed between groups 1 and 2. A correlation between a higher grade of fibrosis and the male sex has been reported in studies on the natural history of liver fibrosis [8,9], but no explanation for this potential association has been provided thus far. It is possible that in these studies such a correlation has been established based on an uncontrolled variable.

Alcohol consumption is a factor that contributes to the progression of liver fibrosis in patients with chronic hepatitis $\mathrm{C}$; however, we found no correlation between alcoholism and a more advanced stage of the disease. Alcohol consumption is a factor difficult to evaluate, and it is possible that, in many cases, true daily ethanol consumption was not reported correctly by the patients, thus underestimating alcohol intake, especially among patients with high consumption. Another explanation is the fact that patients in a more advanced stage of the disease had received recommendations to abstain from alcohol before starting follow-up at our outpatient clinic. The duration of infection, when submitted to logistic regression analysis for the control of the remaining variables, was identified as a variable independently associated with a more advanced stage of the disease. This correlation was predictable since fibrosis develops progressively over time and, therefore, a higher degree of fibrosis was expected for patients with a longer duration of infection.

Considering the importance of the duration of infection for the progression of chronic hepatitis $C$, the index of fibrosis was determined based on the relationship between disease stage and the duration of infection (in years). The median index of fibrosis was 0.14 for the group as a whole. Based on this value, the mean progression for chronic $\mathrm{HCV}$-infected patients with grade 4 disease (cirrhosis) was estimated to be approximately 28.5 years. This result is similar to the 30 years mean time of progression to cirrhosis of estimated by Poynard [8].

Bivariate analysis showed a significant correlation between age at the time of infection and a more advanced stage of the disease $(\mathrm{P}=0.001)$, with this independent association also being observed upon logistic regression analysis. Age at the time of infection was found to be one of the most important host-related factors in the progression of liver fibrosis, as also observed in other studies [8-10].

In order to assess the impact of this variable on the natural history of $\mathrm{HCV}$ infection, the liver fibrosis index was determined according to age group, using a limit of 40 years as a categorization criterion. Patients who were infected after 40 years of age had a higher rate of fibrosis progression $(0.47$ vs $0.11 ; \mathrm{P}<0.001)$.

The mean time of progression to cirrhosis was estimated for each age group based on the median index of fibrosis, with patients infected at less than 40 years of age taking, on average, 36.4 years to develop cirrhosis, while patients infected after 40 years presented a more rapid evolution, with the cirrhosis stage being reached within about 8.5 years (Figure 2). These findings explain why different studies on the natural history of hepatitis $\mathrm{C}$ have shown discrepant results regarding the rate of progression to cirrhosis.

In a study carried out in Ireland on a cohort of young women contaminated through the use of immunoglobulin, only $2 \%$ of the women evolved to cirrhosis within a 17 year follow-up period [2]. Similar findings were reported for 152 women infected through immunoglobulin in Germany [11]. In our study, no patient developed cirrhosis after 15 years of infection. Similarly, Seeffet al. [12] observed liver disease in only $11.8 \%$ of HCV-infected patients after 45 years of infection. In this study, analysis of the causes of mortality after this long period of time revealed that death was attributable to hepatitis $\mathrm{C}$ in only $5.9 \%$ of cases.

Studies conducted on children and adolescents have demonstrated a clinically benign course of HCV infection in this age group [13-15]. These studies reinforce the influence of age on the natural history of 
hepatitis C. On the other hand, studies conducted on older patients contaminated through blood transfusion have reported cirrhosis in about $50 \%$ of cases $[3,16,17]$.

In summary, we conclude that the main factors associated with an advanced stage of chronic hepatitis $\mathrm{C}$ are age at the time of infection and the estimated duration of infection. Patients who acquired HCV after 40 years of age had a higher rate of fibrosis progression, and reached the stage of liver cirrhosis within a period of time which was 4 to 5 times shorter than that observed for patients who were infected at a younger age. These data are important for the clinical followup of patients with hepatitis $C$ and each patient should be submitted to individualized analysis when establishing a disease prognosis, since host-related variables, such as those analyzed here, have a strong impact on the natural history of HCV infection.

\section{References}

1. Villano S.A., Vlahov D., Nelson K..E., et al. Persistence of viremia and the importance of long-term follow-up after acute hepatitis C infection. Hepatology 1999;29(3):908-14.

2. Kenny-Walsh E. Clinical outcomes after hepatitis $\mathrm{C}$ infection from contaminated anti-D immune globulin. Irish Hepatology Research Group. N Engl J Med 1999;340(16):1228-33.

3. Tong M.J., El-Farra N.S., Reikes A.R., Co R.L. Clinical outcomes after transfusion-associated hepatitis C. N Engl J Med 1995;332(22):1463-6.

4. Fattovich G., Giustina G., Degos F., et al. Morbidity and mortality in compensated cirrhosis type $\mathrm{C}$ : a retrospective follow-up study of 384 patients. Gastroenterology 1997;112(2):463-72.

5. Serfaty L., Aumaitre H., Chazouilleres O., et al. Determinants of outcome of compensated hepatitis $\mathrm{C}$ virus-related cirrhosis. Hepatology 1998;27(5):1435-40.

6. Ludwig J. The nomenclature of chronic active hepatitis: an obituary. Gastroenterology 1993;105(1):274-8.

7. Desmet V.J., Gerber M., Hoofnagle J.H., et al. Classification of chronic hepatitis: diagnosis, grading and staging. Hepatology 1994;19(6):1513-20.

8. Poynard T., Bedossa P., Opolon P. Natural history of liver fibrosis progression in patients with chronic hepatitis C. The OBSVIRC, METAVIR, CLINIVIR, and DOSVIRC groups. Lancet 1997;349(9055):825-32.

9. Poynard T., Ratziu V., Charlotte F., et al. Rates and risk factors of liver fibrosis progression in patients with chronic hepatitis C J Hepatol 2001;34(5):730-9.
10. Wong V., Caronia S., Wight D., et al. Importance of age in chronic hepatitis $\mathrm{C}$ virus infection. J Viral Hepat 1997;4(4):255-64.

11. Muller R. The natural history of hepatitis $\mathrm{C}$ : clinical experiences. J Hepatol 1996;24(2):52-4.

12. Seeff L.B., Miller R.N., Rabkin C.S., et al. 45-year followup of hepatitis $\mathrm{C}$ virus infection in healthy young adults. Ann Intern Med 2000;132(2):105-11.

13. Garcia-Monzon C., Jara P., Fernandez-Bermejo M., et al. Chronic hepatitis $\mathrm{C}$ in children: a clinical and immunohistochemical comparative study with adult patients. Hepatology 1998;28(6):1696-701.

14. Hoshiyama A., Kimura A., Fujisawa T., et al. Clinical and histologic features of chronic hepatitis $\mathrm{C}$ virus infection after blood transfusion in Japanese children. Pediatrics 2000; 105 (1 Pt 1):62-5.

15. De Lucas S., Bartolome J., Rodriguez-Inigo E., et al. Distribution of hepatitis $\mathrm{C}$ virus infection in liver biopsies from children and adults with chronic hepatitis C J Med Virol 2001;64(1):1-5.

16. Gordon S.C., Elloway R.S., Long J.C., Dmuchowski C.F. The pathology of hepatitis $\mathrm{C}$ as a function of mode of transmission: blood transfusion vs. intravenous drug use. Hepatology 1993;18(6):1338-43.

17. Yano M., Kumada H., Kage M., et al. The long-term pathological evolution of chronic hepatitis C. Hepatology 1996;23(6):1334-40. 